\title{
Association between the modified Nutrition Risk in Critically III (mNUTRIC) score and clinical outcomes in the intensive care unit: A secondary analysis of a large prospective observational study
}

Na Wang

China Rehabilitation Research Center

Mei-Ping Wang

Capital Medical University

Li Jiang

Xuan Wu Hospital of the Capital Medical University

Bin Du

Peking Union Medical College Hospital

Bo Zhu

Fu Xing hospital

Xiu-Ming Xi ( $\nabla$ xixiuming2937@sina.com )

Fu Xing hospital

\section{Research Article}

Keywords: The modified nutrition risk in critically ill score, Intensive care unit, Mortality

Posted Date: March 16th, 2021

DOl: https://doi.org/10.21203/rs.3.rs-243502/v1

License: (c) This work is licensed under a Creative Commons Attribution 4.0 International License.

Read Full License 
1 Association between the modified Nutrition Risk in Critically Ill (mNUTRIC) score

2 and clinical outcomes in the intensive care unit: A secondary analysis of a large

3 prospective observational study

$4 \mathrm{Na} \mathrm{Wang}^{1^{\#}}$, Mei-Ping Wang ${ }^{2 \#}$, Li Jiang ${ }^{3}$, Bin $\mathrm{Du}^{4}$, Bo Zhu ${ }^{5}$, and Xiu-Ming Xi ${ }^{5 *}$, The Beijing

$5 \quad$ Acute Kidney Injury Trial (BAKIT) workgroup

$6 \quad{ }^{1}$ Emergency department of China rehabilitation research center, Capital Medical University,

7 Beijing, China

$8 \quad{ }^{2}$ Department of Epidemiology and Health Statistics, School of Public Health, Capital Medical

9 University, Beijing, China

$10{ }^{3}$ Department of Critical Care Medicine, Xuan Wu Hospital, Capital Medical University, Beijing,

11 China

$12{ }^{4}$ Medical intensive care unit, Peking Union Medical College Hospital, Peking Union Medical

13 College and Chinese Academy of Medical Sciences, Beijing, China

$14{ }^{5}$ Department of Critical Care Medicine, Fu Xing Hospital, Capital Medical University, Beijing,

15 China

16 \# These authors contributed equally to this work.

17 *Corresponding author: Xiuming Xi, email: xixiuming2937@sina.com

18 Abstract

19 Background

20 Malnutrition in intensive care unit (ICU) patients is associated with adverse clinical outcomes.

21 The nutrition risk in the critically ill score (NUTRIC) was proposed as an appropriate nutritional

22 assessment tool in critically ill patients. This score uses interleukin-6 (IL-6), a biomarker that 
is not always available. This prospective observational study was conducted to identify the nutritional risk in ICU patients using the modified NUTRIC (mNUTRIC) score (which does not include IL-6) and to explore the relationship between 28-day mortality and high mNUTRIC scores.

\section{Methods}

The data were extracted from The Beijing Acute Kidney Injury Trial (BAKIT). This trial was a prospective, observational, multi-centre study conducted in 30 ICUs at 28 tertiary hospitals in Beijing, China, from March 1 to August 31, 2012. In total, 9049 patients were admitted consecutively, and 3107 patients with complete clinical data were included in this study. The predictive capacity of the mNUTRIC score was studied by receiver operating characteristic

33 (ROC) curve analysis. The significance level was set at 5\%.

\section{Results}

Among the 3107 patients, the 28-day mortality rate was $17.4 \%$ (540 patients died). High nutritional risk patients were older $(\mathrm{P}<0.001)$, with higher illness severity scores than low nutritional risk patients. Multivariate analysis revealed that the mNUTRIC score was an independent risk factor for 28-day mortality and mortality increased with increasing scores ( $\mathrm{p}$

$39=0.000)$. The calculated area under curve (AUC) for the mNUTRIC score was 0.763 (CI 0.740 $40-0.786)$

\section{Conclusions}

Nearly $28.2 \%$ of patients admitted to the ICU were at risk of malnutrition, and a high mNUTRIC score was associated with increased ICU length of stay and higher mortality. 


\section{Trial Registration}

This study was registered at www.chictr.org.cn (registration number Chi CTR-ONC-11001875).

Registered on 14 December 2011.

\section{Key words}

The modified nutrition risk in critically ill score, Intensive care unit, Mortality

\section{Background}

Malnutrition is common in intensive care unit (ICU) patients, it is associated with a variety of adverse outcomes, including higher complication rates, prolonged mechanical ventilation, prolonged hospitalization, and higher mortality[1, 2]. For critically ill patients, we need to assess their nutritional status and provide adequate nutritional support[3], so effective tools are needed to determine which ICU patients need nutritional support and the benefits of nutritional support. However, traditional methods of nutrition assessment are limited in the hospital setting. Recently, Heyland et al[4] published the first nutritional risk assessment tool specifically designed for critically ill patients: the NUTRIC score.

The NUTRIC score includs age, the Acute Physiology and Chronic Health Evaluation II (APACHE II) score[5], the Sequential Organ Failure Assessment (SOFA) score[6], comorbidities, days from hospitalization to ICU admission, and the interleukin-6 (IL-6) level, which was developed to link starvation, inflammation, and clinical outcomes[4]. Patients are scored from 0 to 10 , a score of 6 or greater indicates a high nutritional risk.

The NUTRIC score can predict 28-day mortality in a medical-surgical ICU population, highrisk patients who stayed in the ICU for more than 3 days benefited more from nutritional support 
than low-risk patients[4]. But the use of original NUTRIC score is limited by the availability of IL-6, which is not readily available in many institutions. Another study evaluated a modified NUTRIC score excluding IL-6 and found that a score of 5 or higher still indicated a high risk of malnutrition[7]. Moreover, Heyland et al.[4] stated that IL-6 only increased the C-index by 0.007 (from 0.776 to 0.783 ), with no statistical difference. Therefore, they suggested that in settings in which IL-6 is not available, it could be omitted from the NUTRIC score. This adjusted score is called the modified NUTRIC score (mNUTRIC). Rahman et al[8] evaluated this modified NUTRIC score and found that mortality increased by $1.4 \%(95 \% \mathrm{CI}, 1.3-1.5)$ for every point increase in the mNUTRIC score.

Using appropriate nutrition screening and assessment tools will help identify effective strategies that reduce the negative impact of malnutrition. Our study was conducted to identify the prevalence of nutritional risk in general ICU patients based on mNUTRIC scores.

\section{Methods}

\section{Study design and data collection}

This study used a database from a prospective, multi-centre, observational study that investigated the epidemiology of acute kidney injury (AKI) in critically ill patients in 30 ICUs at 28 tertiary hospitals in Beijing, China, from March 1 to August 31, 2012 (the Beijing Acute Kidney Injury Trial (BAKIT)[9]. (for a complete list of these hospitals and the persons responsible for the data acquisition, see Additional file 1). Study subjects included all adult patients (age $\geq 18$ years) admitted consecutively to the ICU. Only the initial ICU admission was considered in this study. The following patients were excluded: patients with preexisting endstage chronic kidney disease, patients already receiving renal replacement therapy (RRT) 
before admission to the ICU, and patients who had received kidney transplantation in the previous 3 months[10]. Pre-existing comorbidities were diagnosed based on the International Classification of Diseases (ICD-10) codes. Patients were followed up until death, until hospital discharge, or for 28 days. Among the 9079 patients who were admitted consecutively, 3107 patients were included in our study (Figure 1).

Thorough follow-up of all patients included in the study was conducted in the first 10 days after ICU admission. The collected data included demographics, anthropometrics, admission diagnosis, comorbidities, daily vital signs and laboratory data, which were used to automatically calculate the APACHE II score, the Simplified Acute Physiology Score II (SAPS II) score[11] and the SOFA score, days from hospital to ICU admission, ICU length of stay (LOS), hospital LOS, use of vasoactive drugs, and length of mechanical ventilation. RRT data were also reported.

The patients were followed up until death, hospital discharge, or for 28 days.

\section{Nutritional support}

Nutritional support methods were based on the guidelines for enteral and parenteral nutrition issued by the European and American Society of Enteroprotective Nutrition[12], combined with our accumulated clinical experience, individualized nutritional support was given to all patients. The patients began enteral nutrition (EN) 20-25 kcal/(kg.d) within $24-48$ hours of admission to the ICU (on average). If the patient was intolerant of EN or had contraindications to EN, parenteral nutrition (PN) support was given within 24 - 48 hours. If EN could not fully meet the nutritional needs of patients, appropriate intravenous supplementation with glucose, amino acids, or fat emulsion was given, that is, the combination of EN and PN. 


\section{Definitions}

110 We used the modified 9-point scale of the NUTRIC score, the mNUTRIC score. We defined

111 the scores from 0 to 4 as "low scores", which indicated a low level of risk of malnutrition, and

112 the scores from 5 to 9 as "high scores", which were associated with worse clinical outcomes.

\section{Statistical analysis}

114 Non-normally distributed continuous variables were expressed as the medians with interquartile

115 ranges (IQRs) and were compared using the Mann-Whitney U test or Kruskal-Wallis analysis

116 of variance with Bonferroni correction. Categorical variables were expressed as the number of

117 cases and proportions and were compared using the Mantel-Haenszel Chi-square test.

118 A multivariate Cox regression analysis was performed using a backward stepwise selection

119 method, with $\mathrm{P}$ value $<0.05$ as the entry criterion, and $\mathrm{P}$ value $\geq 0.10$ as the removal criterion.

120 The assumption of proportional hazards was checked graphically using log (-log (survival

121 probability)) plots and was found to be appropriate. Variables considered for multivariable

122 analysis included age, sex, body mass index (BMI), illness severity scores, use of vasoactive

123 drugs, mechanical ventilation and underlying diseases. We tested for collinearity among all

124 variables using a Cox regression analysis to generate hazard ratios (HR) and 95\% confidence

125 intervals (CIs).

126 Receiver operator characteristic curve analysis was used to calculate the sensitivity and

127 specificity for comparisons of outcomes and mNUTRIC scores. The 28-day survival stratified

128 by low and high mNUTRIC scores was additionally evaluated graphically using the Kaplan-

129 Meier product limit survival plot. 
130 All statistical analyses were performed using SPSS software (IBM Corp., Statistics for

131 Windows, version 22.0, Armonk, NY, USA), with a two-sided $\mathrm{P}$ value $<0.05$ considered

132 statistically significant.

\section{Results}

\section{Study population}

135 Among the 9049 patients enrolled in the BAKIT study, 5942 were excluded for the reasons

136 shown in Figure 1, leaving 3107 patients for analysis. The characteristics of the entire cohort

137 are shown in Table 1. The median age was 64.0 (IQR: $51.0-77.0$ ) years, and $61.5 \%$ were men.

138 The all-cause 28-day mortality rate was $17.4 \%$ and the median ICU LOS was 4.0 (IQR: 2.0 -

139 9.0) days. Among the included patients, the median BMI was 24.0 (IQR: $21.0-26.0$ ) kg/m²,

140 the median APACHE II score was 14.0 (IQR:10.0 - 20.0), the median SOFA score was 6.0

141 (IQR: 3.0 - 8.0), and the median number of comorbidities was 1 (IQR: 0 - 2). Mechanical

142 ventilation was used in $2021(65.0 \%)$ patients, 1307 patients $(42.1 \%)$ received vasopressors,

143 and 281 patients $(9.0 \%)$ underwent RRT. A total of 876 patients $(28.2 \%)$ had high mNUTRIC

144 scores.

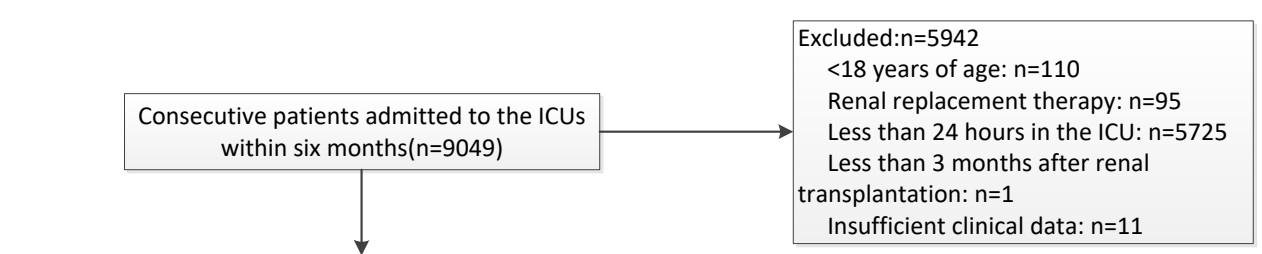

Figure.1 Flowchart of validation cohort 
Table 1 Patient characteristics by mNUTRIC score

\begin{tabular}{|c|c|c|c|c|}
\hline Characteristic & $\begin{array}{l}\text { All patients } \\
(\mathrm{n}=3107) \\
\text { Median(IQR) } \\
\text { Number }(\%)\end{array}$ & $\begin{array}{l}\text { Low nutrition } \\
\text { risk(n=2231) } \\
\text { Median(IQR) } \\
\text { Number (\%) }\end{array}$ & $\begin{array}{l}\text { High nutrition } \\
\text { risk(n=876) } \\
\text { Median(IQR) } \\
\text { Number (\%) }\end{array}$ & $\begin{array}{c}\mathbf{P} \\
\text { value }\end{array}$ \\
\hline Age(years) & $64(51-77)$ & $60(47-72)$ & $76(66-82)$ & $<0.001$ \\
\hline Male sex & $1912(61.5)$ & $1378(61.8)$ & $534(61.0)$ & 0.919 \\
\hline BMI & $24(21-26)$ & $24(22-26)$ & $23(21-26)$ & 0.003 \\
\hline Vasoactive therapy & $1307(42.1)$ & $954(42.8)$ & $353(40.3)$ & 0.457 \\
\hline Mechanical ventilation & $2021(65.0)$ & $1354(60.7)$ & $667(76.1)$ & $<0.001$ \\
\hline Sepsis & $896(28.8)$ & $419(18.8)$ & $477(54.5)$ & $<0.001$ \\
\hline \multicolumn{5}{|l|}{ Severity of illness } \\
\hline APACHEII & $14(10-20)$ & $12(8-15)$ & $23(19-28)$ & $<0.001$ \\
\hline SAPSII & $34(26-45)$ & $30(23-38)$ & $50(39-64)$ & $<0.001$ \\
\hline SOFA & $6(3-8)$ & $4(3-7)$ & $9(6-11)$ & $<0.001$ \\
\hline NUTRIC score & $3(2-5)$ & $3(2-3)$ & $6(5-7)$ & $<0.001$ \\
\hline \multicolumn{5}{|l|}{ Admission category } \\
\hline medical & $1480(47.6)$ & $878(39.4)$ & $602(68.7)$ & \\
\hline surgical & $1627(52.4)$ & $1353(60.6)$ & $274(31.3)$ & \\
\hline \multicolumn{5}{|l|}{ Comorbid diseases } \\
\hline Cancer & $486(15.6)$ & $297(13.3)$ & $189(21.6)$ & \\
\hline Hypertension & $1222(39.3)$ & $739(33.1)$ & $483(55.1)$ & \\
\hline Coronary disease & $615(19.8)$ & $293(13.1)$ & $322(36.8)$ & \\
\hline Chronic kidney disease & $170(5.5)$ & $63(2.8)$ & $107(12.2)$ & \\
\hline Diabetes & $532(17.1)$ & $277(12.4)$ & $255(29.1)$ & \\
\hline COPD & $166(5.3)$ & $89(4.0)$ & $77(8.8)$ & \\
\hline \multicolumn{5}{|l|}{$\begin{array}{l}\text { Category of ICU } \\
\text { admission } \\
\text { diagnosis }\end{array}$} \\
\hline Cardiovascular & $848(27.3)$ & $681(30.5)$ & $167(19.1)$ & \\
\hline Respiratory & $548(17.6)$ & $316(14.2)$ & $232(26.5)$ & \\
\hline
\end{tabular}




\begin{tabular}{|c|c|c|c|c|}
\hline Neurologic & $462(14.9)$ & $321(14.4)$ & $141(16.1)$ & \\
\hline Trauma & $238(7.7)$ & 191(8.6) & $47(5.4)$ & \\
\hline Gastrointestinal & $607(19.4)$ & $413(18.5)$ & $194(22.1)$ & \\
\hline Metabolic & $77(2.5)$ & $43(1.9)$ & $34(3.9)$ & \\
\hline \multicolumn{5}{|l|}{ Outcome data } \\
\hline ICU LOS(days) & $4(2-9)$ & $4(2-7)$ & $6(3-13)$ & $<0.001$ \\
\hline Hospital LOS(days) & $19(12-29)$ & $19(12-28)$ & $21(11-34)$ & 0.002 \\
\hline 28-day mortality & $540(17.4)$ & $208(9.3)$ & $332(37.9)$ & $<0.001$ \\
\hline In-hospital mortality & $521(16.8)$ & $173(7.8)$ & $348(39.7)$ & $<0.001$ \\
\hline AKI & $1334(42.9)$ & $752(33.7)$ & $582(66.4)$ & $<0.001$ \\
\hline RRT & $281(9.0)$ & $108(4.8)$ & $173(19.7)$ & $<0.001$ \\
\hline \multicolumn{5}{|l|}{ Hospitalization } \\
\hline expense ( thousand & $40(19-96)$ & $34(17-87)$ & $55(27-113)$ & $<0.001$ \\
\hline
\end{tabular}

Data are expressed as the median (interquartile range), and number (percentage). BMI,body mass index;SAPS II, Simplified Acute Physiology Score II; SOFA, Sequential Organ Failure Assessment; APACHE II, Acute Physiology and Chronic Health Evaluation II; NUTRIC score, the nutrition risk in the critically ill score;COPD, chronic obstructive pulmonary disease;LOS, length of stay;AKI, acute kidney injury; RRT, renal replacement therapy.

\section{Characteristics of high nutritional risk patients}

155 From Table 1, we can see high nutritional risk patients were older $(\mathrm{P}<0.001)$, with higher illness

156 severity scores than low nutritional risk patients. High nutritional risk patients were more likely

157 to present with sepsis on ICU admission and had longer durations of ICU and hospital stays

158 when compared to the low nutritional risk group. Furthermore, mechanical ventilation was

159 more commonly used in high nutritional risk patients $(76.1 \%$ vs $60.7 \%$; $\mathrm{P}<0.001)$. The 28 -day

160 mortality and in-hospital mortality rates were higher among high nutritional risk patients than

161 low nutritional risk patients $(\mathrm{P}<0.001)$. 
164 2), and the 28-day mortality for the maximum mNUTRIC score was $67.4 \%$.

165 mNUTRIC score, sepsis and AKI were independent predictors of 28-day mortality. The

Figure 2. The 28-day mortality according to modified NUTRIC score.

\section{High mNUTRIC score and the 28-day mortality}

In multivariate Cox regression analysis (Table 2), after adjusting for age, sex, BMI, illness severity scores, use of vasoactive drugs, mechanical ventilation and underlying diseases, the presence of high mNUTRIC scores was associated with a higher risk of mortality (Figure 3).

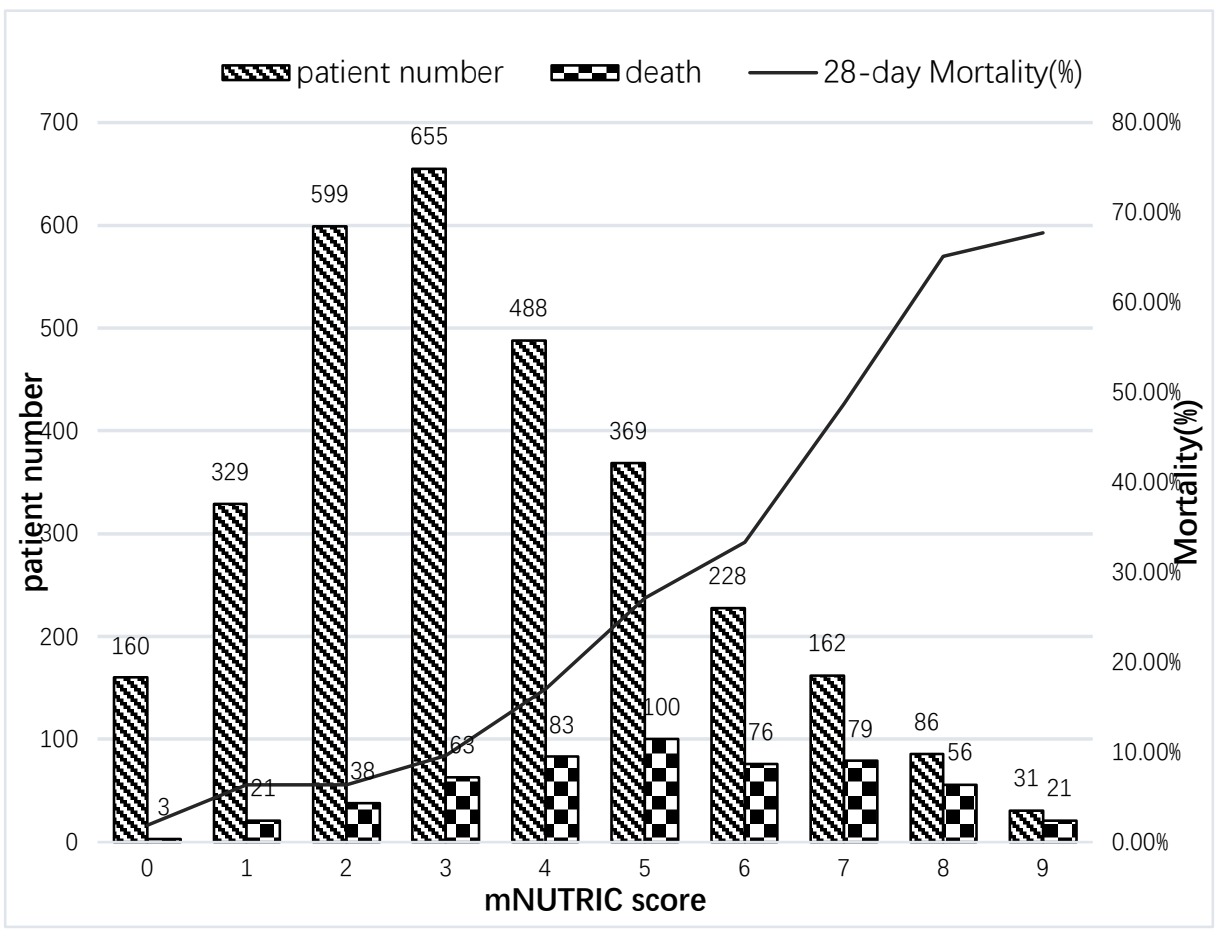

Table 2 Multivariate Cox regression analysis of 28-day mortality in all patients

\begin{tabular}{lccc}
\hline Characteristic & Hazard ratio & $\mathbf{9 5 \% C I}$ & $\mathbf{P}$ \\
\hline mNUTRIC score & 1.430 & $1.351-1.514$ & 0.000 \\
Sepsis & 2.832 & $2.272-3.529$ & 0.000 \\
AKI & 2.171 & $1.732-2.720$ & $<0.001$ \\
\hline
\end{tabular}


173 mNUTRIC score, the modified nutrition risk in the critically ill score; AKI, acute kidney

174 injury; $\mathrm{CI}$,confidence interval

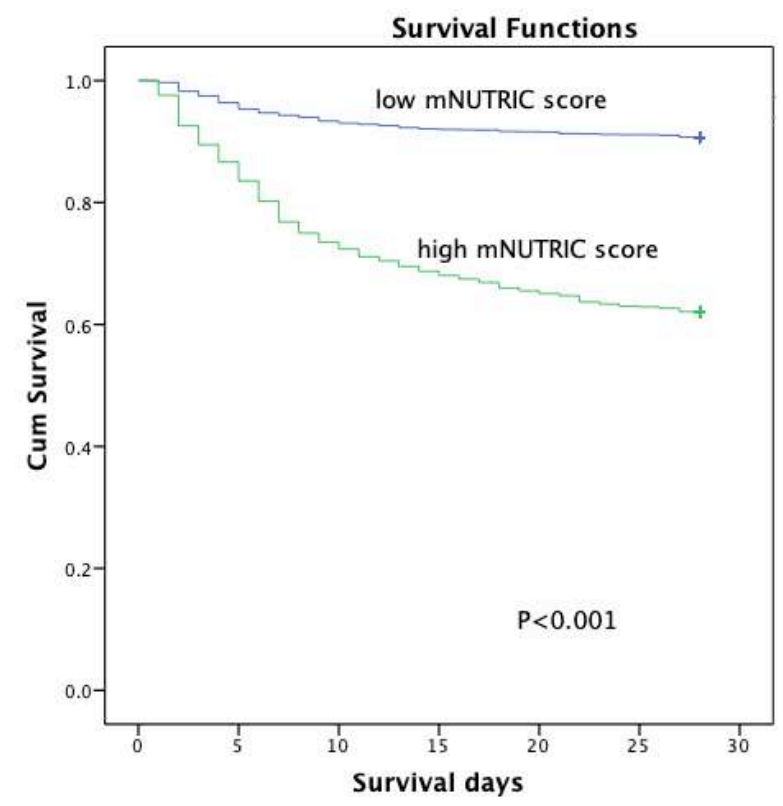

175 Figure 3 Survival curve of 28-day mortality stratified by mNUTRIC scores.

176 Area under the curve of scores for predicting 28-day mortality

177 We can see that in this cohort and each subgroup, the areas under the curve (AUCs) of the

178 mNUTRIC score for predicting 28-day mortality indicated good predictive performance of the

179 score (Figure 4). In the ROC curve for the mNUTRIC score, the best cut-off value was at 4

180 (sensitivity $61.48 \%$ and specificity $78.81 \%$ ) in this cohort, and the Youden index was 0.4029. 

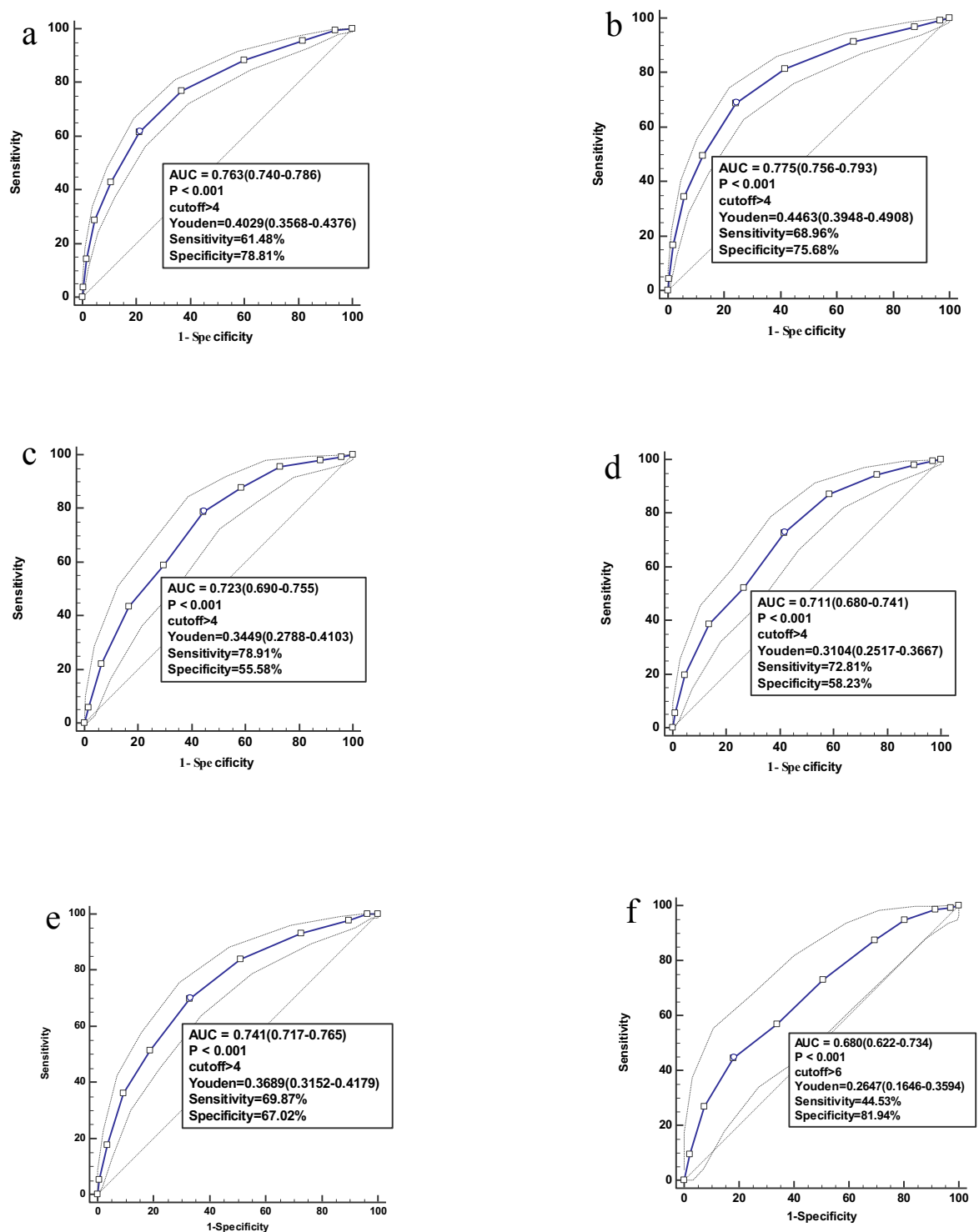

182 Figure 4 Performance of mNUTRIC scores in predicting 28-day mortality. a. All patients (n=3107); b. All 183 mechanical ventilation patients $(n=2021)$; c. Medical mechanical ventilation patients $(n=751)$; d. Sepsis 184 patients $(\mathrm{n}=896)$; e. AKI patients $(\mathrm{n}=1334)$; f. CRRT patients $(\mathrm{n}=281)$

\section{Discussion}

This study was a secondary analysis of a prospective observational study in surgical-medical ICUs. We used a validated nutrition assessment tool in an attempt to demonstrate an association patients, and malnutrition was associated with a poor prognosis. 
191 In the present study, $28.2 \%$ of the critically ill patients admitted to the ICU were at high 192 nutritional risk and had mNUTRIC scores $\geq 5$. These findings were similar to the results of a 193 study conducted in Turkey[13], in which $22.4 \%$ patients were evaluated as having high scores 194 (between 5 and 9). Lew et al[14] also demonstrated that the prevalence of malnutrition in the 195 ICU was 28\% using the 7-point Subjective Global Assessment (7-point SGA) to determine 196 patients' nutritional status. Recently, a study[15] reported that $45 \%$ of mechanically ventilated 197 patients admitted to the ICU were at high nutritional risk. Similarly, Kalaiselvan et al.[16] 198 reported that $42.5 \%$ of mechanically ventilated patients had NUTRIC scores $\geq 5$. Our study is 199 more generalizable because of the inclusion of both medical and surgical patients. The 200 aforementioned studies included only patients on mechanical ventilation, and patients on 201 mechanical ventilation were more seriously ill than those not on mechanical ventilation. The 202 differences among studies are mainly the result of different populations and nutrition screening 203 tools.

204 In our study, the 28-day mortality associated with the maximum mNUTRIC score was $67.7 \%$, 205 which is similar to the finding in the study by Jeong[17], in which this rate was $62.5 \%$. 206 Compared with patients with a low NUTRIC score, patients with high NUTRIC score had a 207 higher mortality rate and longer ICU LOS. Similar results were reported by Mendes et al[1], in 208 which the NUTRIC score was used in an ICU population, and the findings are consistent with 209 those in the study by Heyland[4].

210 The mortality rate in our study was $17.4 \%$, which was lower than the rate reported in the second 211 validation study of the NUTRIC score (29\%) by Rahman et al[8]. This difference may be 
because our study included many postoperative care patients. In this study, we found that the

213 mNUTRIC score was a good prognostic predictor in critically ill patients and that high

214 mNUTRIC scores were associated with an elevated risk of death at 28 days $(\mathrm{HR}=1.430,95 \%$

$215 \mathrm{CI}=1.351$ to $1.514, \mathrm{P}=0.000)$. This finding is consistent with those of prior studies $[1,18,19]$.

216 Several studies have shown that the beneficial effects of adequate nutritional support are more

217 evident in high-risk patients than in low-risk patients[20, 21]. The mNUTRIC score may be

218 helpful in guiding clinicians in providing adequate nutritional support to ICU patients.

219 The mNUTRIC score was found to have a fair predictive performance for 28-day mortality in 220 this cohort (AUC 0.763; 95\% CI 0.740 - 0.786) and each subgroup. These results are in line 221 with those of the initial validation study by Heyland et al. (AUC: 0.783)[4] and a recently 222 published validation study of the mNUTRIC score by Mukhopadhyay et al. (AUC 0.71)[22]. 223 Recently, a study[17] showed that the AUC of the NUTRIC score for the prediction of 28-day 224 mortality was 0.762 (95\% CI: $0.718-0.806)$, while that of the mNUTRIC score was 0.757 (95\% 225 CI: 0.713-0.801). There was no significant difference between the two scores $(\mathrm{p}=0.45)$. The 226 mNUTRIC score is a good nutritional risk assessment tool for critically ill patients.

227 We found that the best cut-off value for the mNUTRIC score was $>4$ (sensitivity $61.48 \%$ and 228 specificity $78.81 \%$ ) in this cohort, and the Youden index was 0.4029 , which is consistent with 229 previous work by de Vries et al[23]. However, in another study, the best cut-off value was at 6 230 (sensitivity $75 \%$ and specificity $65 \%$ ), and the Youden index was 0.401 [17]. Jung et al reported 231 that patients were considered to be at high risk of malnutrition and to benefit from aggressive 232 nutritional support when their mNUTRIC score was $\geq 5$ [24]. Our study included patients with 
various diseases, while Jung's study population was limited to patients with sepsis. Further

234 investigation is needed to find the best cut-off value of the mNUTRIC score to define the high-

235 risk group.

236 The limitations of our study stem mainly from the fact that it is a secondary analysis of an

237 original database that lacked data on inflammation indicators such as IL-6. Therefore, we could

238 not calculate the NUTRIC score to verify the differences between the two scores. Second,

239 nutrition history and feeding parameters were not available in our cohort, so the associations

240 among nutritional adequacy, mNUTRIC score and mortality could not be confirmed by our

241 results.

\section{Conclusion}

243 Patients were considered to be at high risk of malnutrition when their mNUTRIC score was >

244 4. The mNUTRIC score is a practical, easy-to-use tool based on variables that are easy to obtain 245 in the critical care setting.

\section{Abbreviations}

247 ICU: intensive care unit;NUTRIC: the nutrition risk in the critically ill score;IL-6: interleukin248 6;mNUTRIC: the modified nutrition risk in the critically ill score;ROC: receiver operating 249 characteristic;AUC: area under curve;AKI: acute kidney injury;BAKIT: the Beijing Acute 250 Kidney Injury Trial;BMI: body mass index;RRT: renal replacement therapy;APACHE II: acute 251 physiology and chronic health evaluation II;SAPS II: the simplified acute physiology score 252 II;SOFA: sequential organ failure assessment;LOS: length of stay;EN: enteral nutrition; PN: 
253 parenteral nutrition; IQR: interquartile range;HR: hazard ratio; CI: confidence interval;COPD:

254 chronic obstructive pulmonary disease

255 Acknowledgments

256 The authors thank all members of the Beijing Acute Kidney Injury Trial (BAKIT) work group

257 (see Additional file 1) for participating in database management.

\section{Authors' contributions}

259 NW and MPW designed and carried out the study, NW performed the statistical analysis, and 260 drafted the manuscript. LJ and BD were involved in design and in acquisition of data and helped 261 to revise the manuscript critically for important content. BZ was involved in the design and the 262 statistical analysis. The Beijing Acute Kidney Injury Trial (BAKIT) Workgroup participated in 263 acquisition and interpretation of data. XX conceived of the study, participated in its design, and 264 helped to revise manuscript. All authors read and approved the final manuscript.

\section{Conflict of interest}

266 The authors declare that they have no conflict of interest.

\section{$267 \quad$ Funding}

268 The study was supported by a grant from the Beijing Municipal Science \& Technology 269 Commission, a government fund used to improve health-care quality (No. D101100050010058).

270 It offered financial support for data collection.

\section{$271 \quad$ Availability of data and materials}

272 The datasets used and analyzed during the current study are available from the corresponding 273 author on reasonable request. 
275 This study was approved by the Institutional Review Boards of the Ethics Committees of the

276 lead study centre (Fu Xing Hospital, Capital Medical University, China) and all other

277 participating hospitals (Additional file 2). We confirm that all methods were carried out in

278 accordance with relevant guidelines and regulations.

279 Being an observational study, written informed consent from participants to partake into the 280 study was not necessary. Hence, we obtained an informed consent waiver from the above 281 ethical committees.

\section{Consent for publication}

283 Not applicable.

\section{Competing interests}

285 The authors declare that they have no competing interests.

\section{Reference}

287 1. Correia MI, Waitzberg DL. The impact of malnutrition on morbidity, mortality, length 288 of hospital stay and costs evaluated through a multivariate model analysis. Clin $289 \quad$ Nutr 2003;22(3):235-239.

290 2. Heyland DK, Cahill N, Day AG. Optimal amount of calories for critically ill patients: 291 depends on how you slice the cake! Crit Care Med 2011;39(12):2619-26.

292 3. Al-Dorzi HM, Albarrak A, Ferwana M,et al. Lower versus higher dose of enteral caloric 293 intake in adult critically ill patients: a systematic review and meta-analysis. Crit Care 2016;20(1):358. 
4. Heyland DK, Dhaliwal R, Jiang X,et al. Identifying critically ill patients who benefit the most from nutrition therapy: the development and initial validation of a novel risk assessment tool. Crit Care 2011;15(6):R268.

5. Knaus WA, Draper EA, Wagner DP,et al. APACHE II: a severity of disease classification system. Crit Care Med 1985;13(10):818-29.

6. Vincent JL, de Mendonca A, Cantraine F,et al. Use of the SOFA score to assess the incidence of organ dysfunction/failure in intensive care units: Results of a multicenter, prospective study. Critical Care Medicine 1998;26(11):1793-1800.

7. Heyland DK, Dhaliwal R, Wang M,et al. The prevalence of iatrogenic underfeeding in the nutritionally 'at-risk' critically ill patient: Results of an international, multicenter, prospective study. Clin Nutr 2015;34(4):659-66.

8. Rahman A, Hasan RM, Agarwala R,et al. Identifying critically-ill patients who will benefit most from nutritional therapy: Further validation of the "modified NUTRIC" nutritional risk assessment tool. Clin Nutr 2016;35(1):158-62.

9. Luo X, Jiang L, Du B, Wen Y,et al. A comparison of different diagnostic criteria of acute kidney injury in critically ill patients. Crit Care 2014; 18(4):R144.

311 10. Piccinni P, Cruz DN, Gramaticopolo S, et al. Prospective multicenter study on epidemiology of acute kidney injury in the ICU: a critical care nephrology Italian collaborative effort (NEFROINT). Minerva Anestesiol 2011;77(11):1072-83. 
314 11. Le Gall JR, Lemeshow S, Saulnier F. A new Simplified Acute Physiology Score (SAPS II) based on a European/North American multicenter study. JAMA 1993;270(24):295763.

12. McClave SA, Taylor BE, Martindale RG, et al. Guidelines for the Provision and Assessment of Nutrition Support Therapy in the Adult Critically Ill Patient: Society of Critical Care Medicine (SCCM) and American Society for Parenteral and Enteral Nutrition (A.S.P.E.N.). JPEN J Parenter Enteral Nutr 2016; 40(2):159-211.

13. Ozbilgin S, Hanci V, Omur D, et al.Morbidity and mortality predictivity of nutritional assessment tools in the postoperative care unit. Medicine (Baltimore) 2016;95(40):e5038.

14. Lew CCH, Wong GJY, Cheung KP,et al. Association between Malnutrition and 28-Day Mortality and Intensive Care Length-of-Stay in the Critically ill: A Prospective Cohort Study. Nutrients 2017;10(1).

15. Ata Ur-Rehman HM, Ishtiaq W, Yousaf M, et al.Modified Nutrition Risk in Critically Ill (mNUTRIC) Score to Assess Nutritional Risk in Mechanically Ventilated Patients: A Prospective Observational Study from the Pakistani Population. Cureus

16. Kalaiselvan M, Renuka M, Arunkumar A .Use of nutrition risk in critically ill (nutric) score to assess nutritional risk in mechanically ventilated patients: A prospective observational study. Indian J Crit Care Med 2017;21(5):253-56. 
334 17. Jeong DH, Hong SB, Lim CM, et al. Comparison of Accuracy of NUTRIC and Modified NUTRIC Scores in Predicting 28-Day Mortality in Patients with Sepsis: A Single Center Retrospective Study. Nutrients 2018;10(7).

18. Mendes R, Policarpo S, Fortuna P,et al. Portuguese NUTRIC Study Group. Nutritional risk assessment and cultural validation of the modified NUTRIC score in critically ill patients - A multicenter prospective cohort study. J Crit Care 2017;37:249.

19. Oliveira ML, Heyland DK, Silva FM, et al. Complementarity of modified NUTRIC score with or without C-reactive protein and subjective global assessment in predicting mortality in critically ill patients. Rev Bras Ter Intensiva 2019;31(4):490-496.

20. Alberda $\mathrm{C}$, Gramlich L, Jones $\mathrm{N}$ et al.The relationship between nutritional intake and clinical outcomes in critically ill patients: results of an international multicenter observational study. Intensive Care Med 2009;35:1728-1737.

21. Artinian V, Krayem H, DiGiovine B. Effects of early enteral feeding on the outcome of critically ill mechanically ventilated medical patients. Chest 2006;129:960-967.

22. Mukhopadhyay A, Henry J, Ong V et al. Association of modified NUTRIC score with 28-day mortality in critically ill patients. Clin Nutr 2017;36(4):1143-1148.

23. de Vries MC, Koekkoek WK, Opdam MH et al. Nutritional assessment of critically ill patients: validation of the modified NUTRIC score. Eur J Clin Nutr 2018;72:428-435. 
353 24. Jung YT, Park JY, Jeon J et al. Association of Inadequate Caloric Supplementation with 30-Day Mortality in Critically Ill Postoperative Patients with High Modified NUTRIC Score. Nutrients 2018;10:1589.

356 Table 1 Patient characteristics by mNUTRIC score

357 Table 2 Multivariate Cox regression analysis of 28-day mortality in all patients

358 Figure.1 Flowchart of validation cohort

359 Figure 2. The 28-day mortality according to modified NUTRIC score.

360 Figure 3 Performance of mNUTRIC scores in predicting 28-day mortality. a. All patients

361 ( $\mathrm{n}=3107)$; b. All mechanical ventilation patients $(\mathrm{n}=2021)$; c. Medical mechanical ventilation

362 patients $(n=751)$; d. Sepsis patients $(n=896)$; e. AKI patients $(n=1334)$; f. CRRT patients

$363 \quad(\mathrm{n}=281)$

364 Figure 4 Survival curve of 28-day mortality stratified by mNUTRIC scores. 
Figures

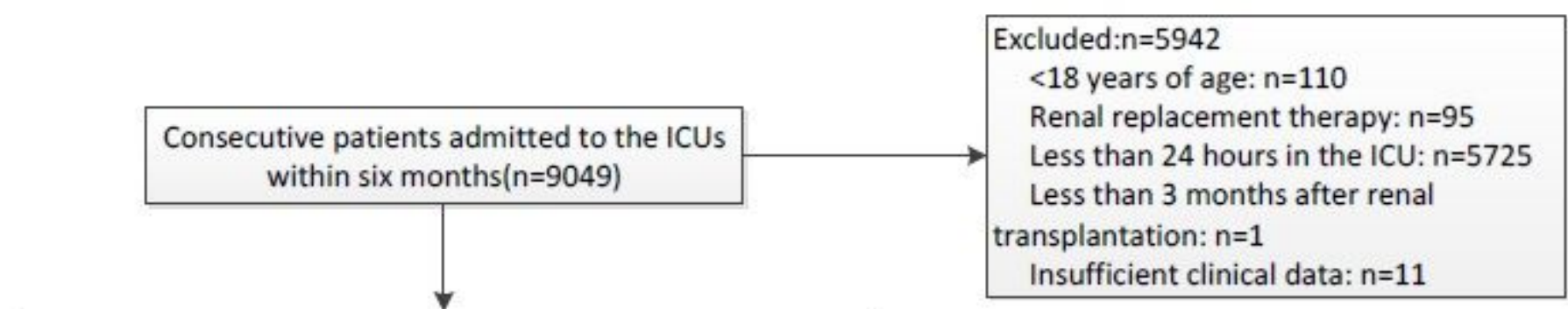

Consecutive patients after preliminary screening $(n=3107)$

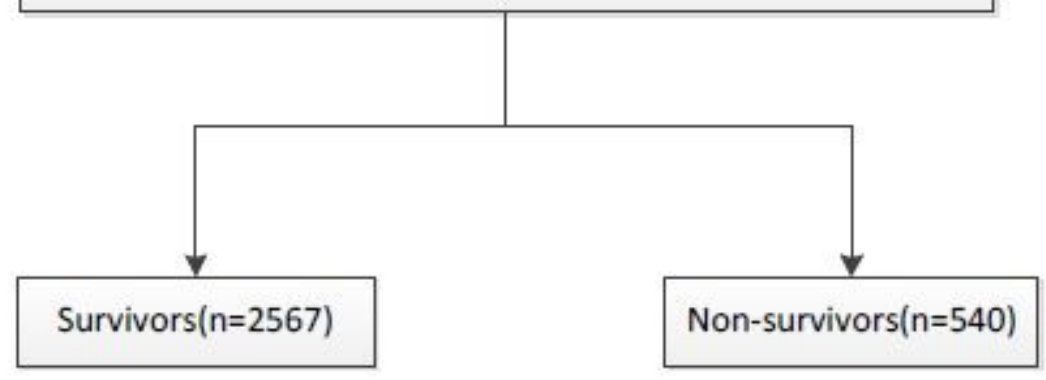

Figure 1

Flowchart of validation cohort 


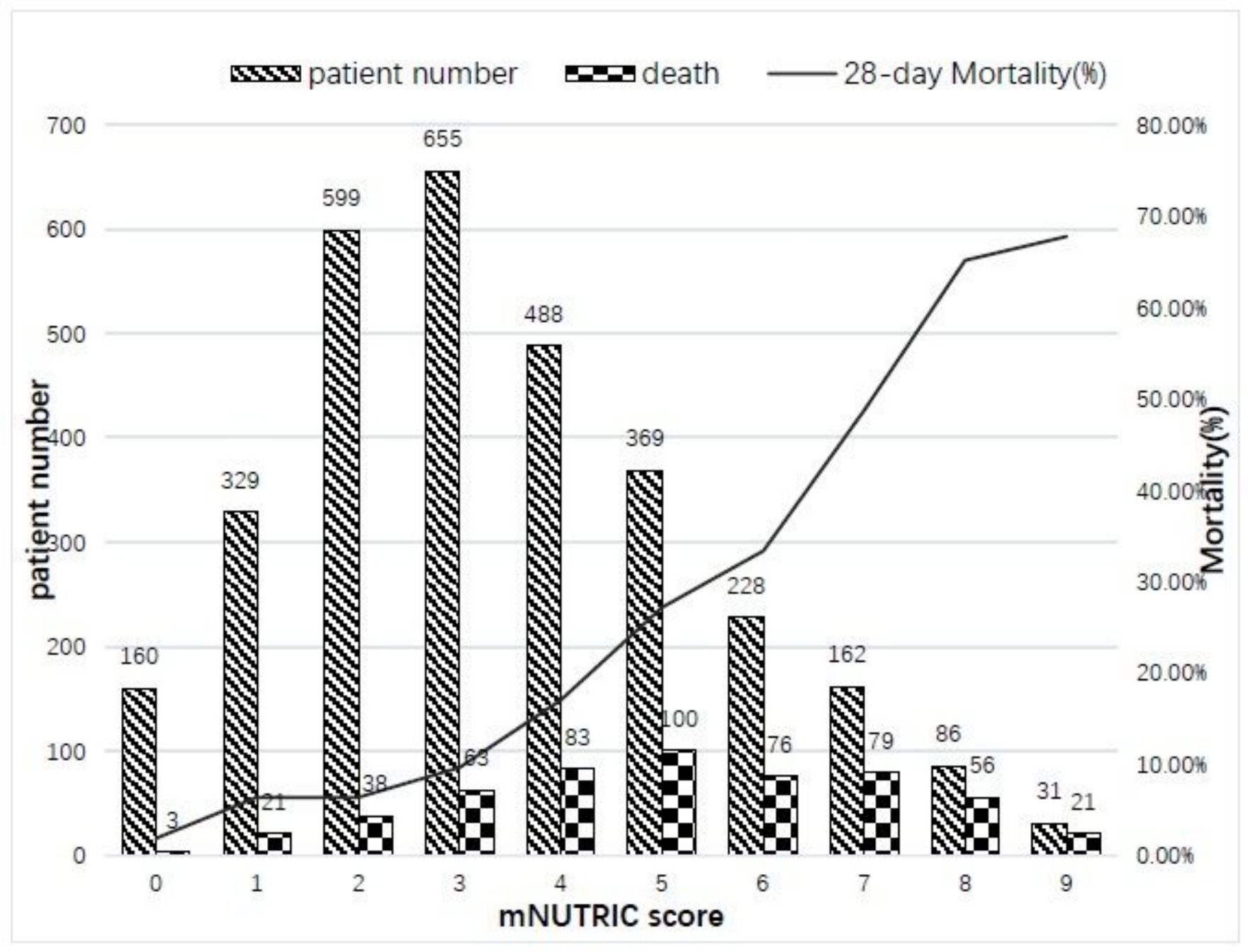

Figure 2

The 28-day mortality according to modified NUTRIC score. 


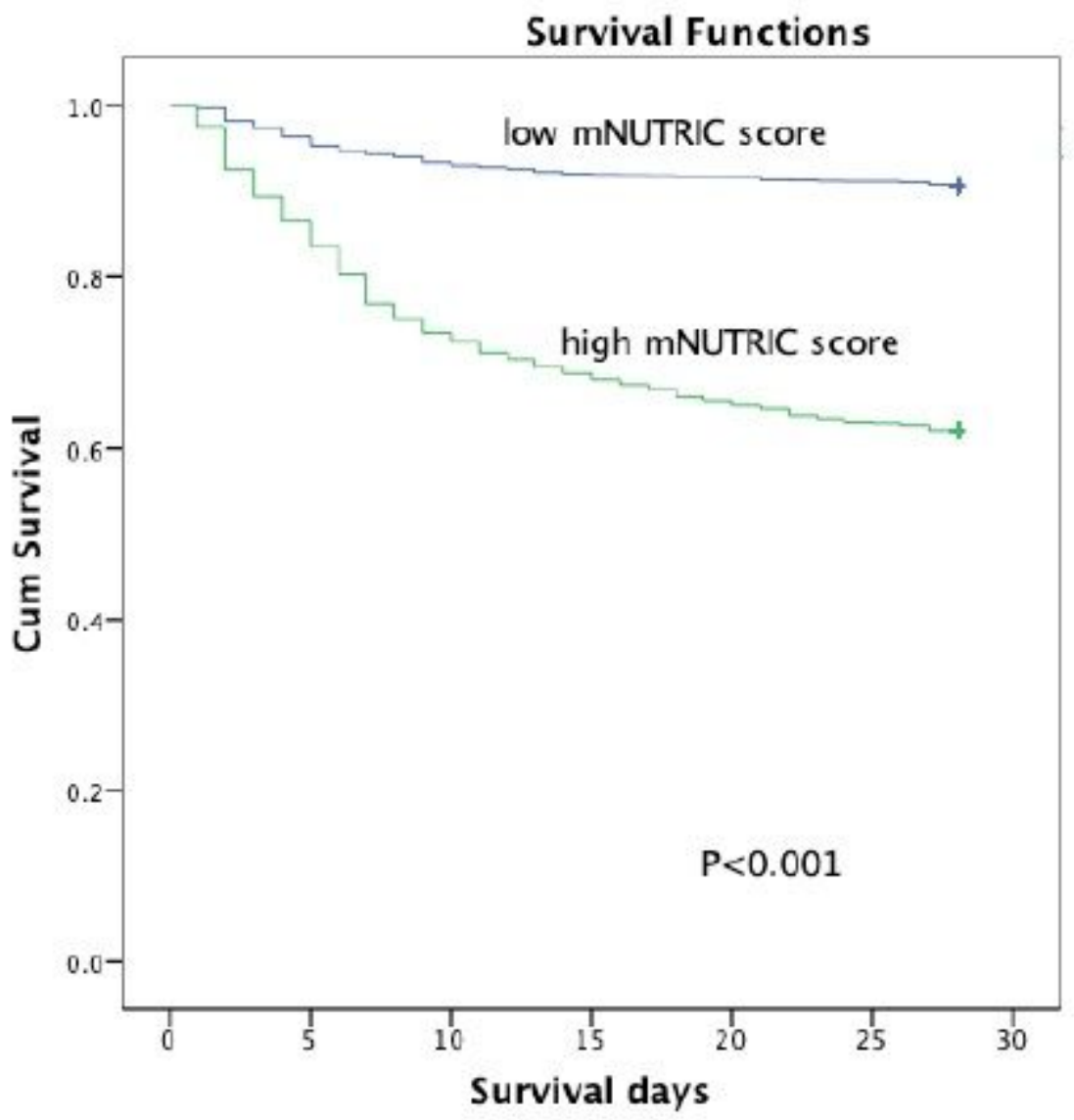

Figure 3

Survival curve of 28-day mortality stratified by mNUTRIC scores. 

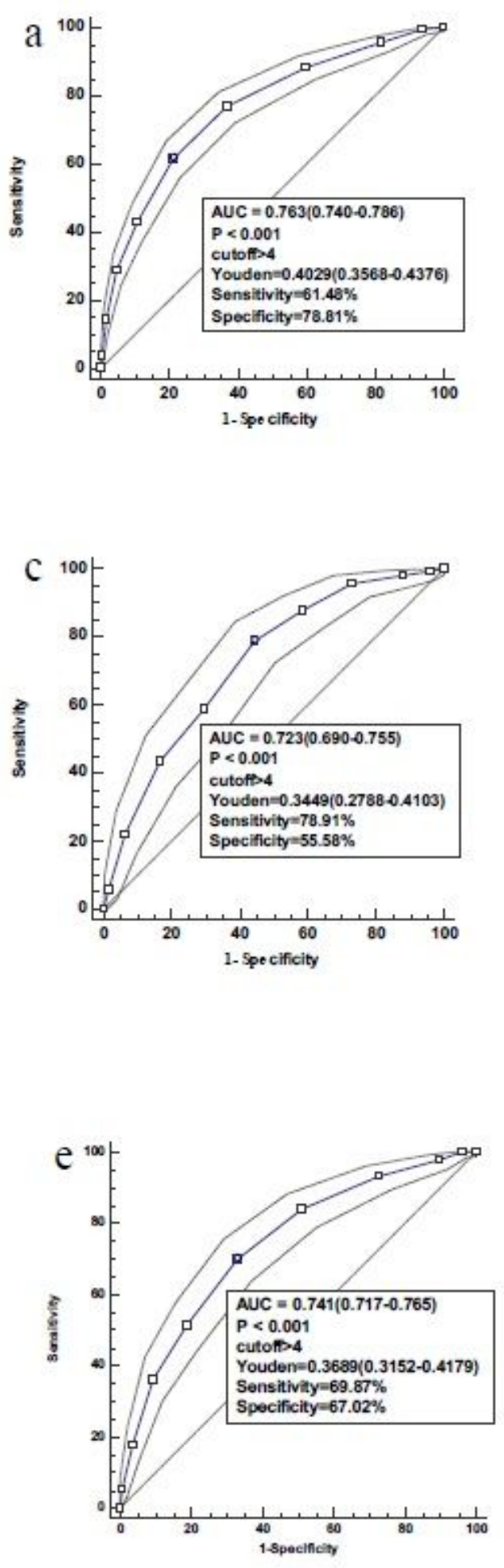
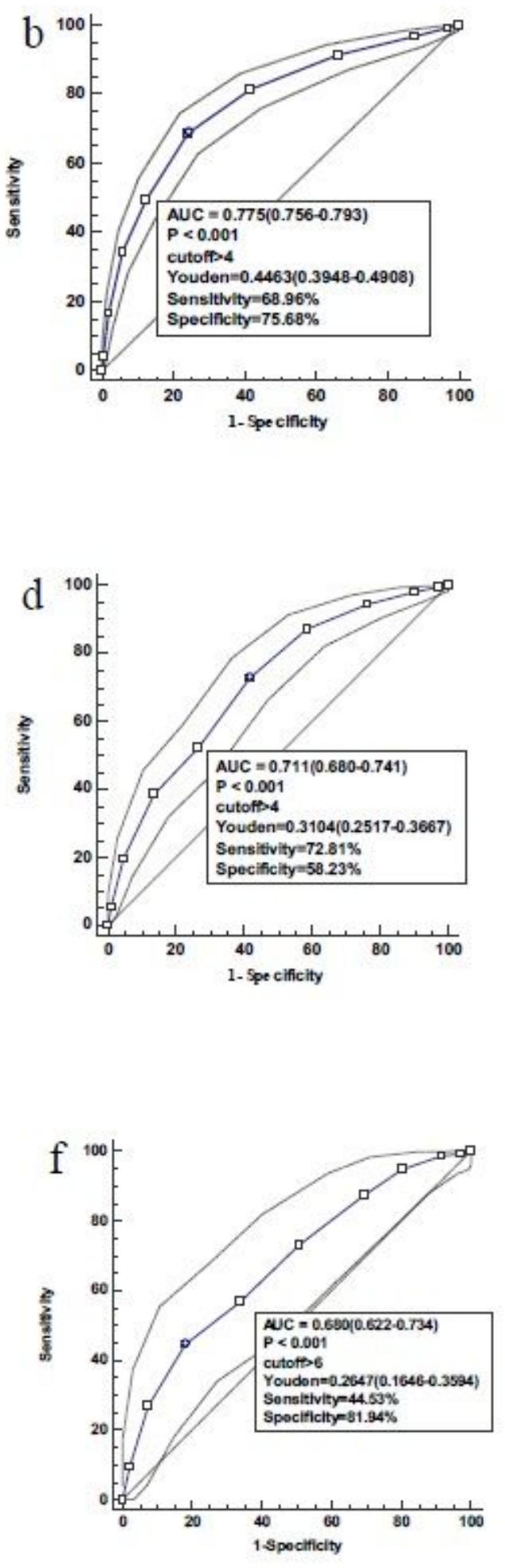

\section{Figure 4}

Performance of mNUTRIC scores in predicting 28-day mortality. a. All patients $(n=3107) ; b$. All mechanical ventilation patients $(n=2021)$; c. Medical mechanical ventilation patients $(n=751)$; d. Sepsis patients $(n=896)$; e. AKI patients $(n=1334) ;$ f. CRRT patients $(n=281)$

\section{Supplementary Files}


This is a list of supplementary files associated with this preprint. Click to download.

- SupplementaryMaterial.docx 